\title{
CONCENTRACIÓN DE INMUNOGLOBULINAS TOTALES EN CALOSTROS DE VACAS EN EXPLOTACIONES LECHERAS DE COSTA RICA ${ }^{1}$
}

\author{
Jorge Alberto Elizondo-Salazar ${ }^{2}$
}

\begin{abstract}
RESUMEN
Concentración de inmunoglobulinas totales en calostros de vacas en explotaciones lecheras de Costa Rica. El objetivo del presente estudio fue determinar la concentración de inmunoglobulinas (Igs) totales en el calostro producido por vacas lecheras y establecer el efecto que la raza y el número de parto pueden tener sobre dicha concentración. Los datos corresponden a determinaciones de concentración de Igs totales determinadas por medio de un calostrómetro en 537 muestras obtenidas entre los años 2010 y 2012, durante los meses de agosto a noviembre en 50 fincas lecheras ubicadas en las provincias de San José, Alajuela, Heredia y Cartago. Las razas de las vacas se clasificaron en Holstein, Jersey, cruce HolsteinxJersey y otras. El tamaño de las fincas osciló entre 10 y 300 vacas en ordeño. Para fines del presente estudio, se consideró un calostro de buena calidad cuando la concentración de Igs fue $\geq 50 \mathrm{mg} /$ ml. La concentración de Igs osciló entre 10 y 140 con un promedio de $85 \mathrm{mg} / \mathrm{ml}$. Del total de muestras analizadas, $13,2 \%$ presentaron una concentración inadecuada de Igs. Al considerar el efecto de raza sobre la concentración de Igs, no se encontraron diferencias significativas. El efecto de número de parto de la vaca influyó significativamente sobre la concentración de Igs encontrándose que a mayor número de parto la concentración de Igs aumenta. La información del estudio indica que la calidad de calostro es alta y que una falla en la transferencia de inmunidad pasiva podría deberse a factores como el volumen del calostro consumido, y el tiempo que transcurre desde el nacimiento y la primera toma de calostro.
\end{abstract}

Palabras clave: terneras, ganado de leche, salud animal, inmunidad pasiva.

\begin{abstract}
Total immunoglobulin concentration in colostrum produced by dairy cows in Costa Rica. The objective of this study was to determine the concentration of total immunoglobulins (Ig) in colostrum produced by dairy cows and establish the effect that breed and parity can have on Ig concentration. The data presented correspond to determinations of concentration of total Ig determined by a colostrometer in 537 colostrum samples obtained between the year 2010 and 2012, during the months of August to November in 50 dairy farms in the provinces of San José, Alajuela, Heredia and Cartago. Cow breeds were classified into Holstein, Jersey, HolsteinxJersey and other. Farm size ranged from 10 to 300 milking cows. For the purposes of this study, good quality colostrum was considered when Ig concentration was $\geq 50 \mathrm{mg} / \mathrm{ml}$. Immunoglobulin concentration ranged from 10 to 140 with an average of 85 $\mathrm{mg} / \mathrm{ml}$. Of all the samples analyzed, $13.2 \%$ had an inadequate concentration of Ig. When considering breed effect on Ig concentration, no significant differences were found. Parity number significantly influenced Ig concentration and it was found that Ig concentration increases linearly with parity number. The information collected in this study indicates that Ig concentration in colostrum is good and that failure of passive transfer of immunity could be due to other factors such as the volume of colostrum consumed and the time between birth and the first feeding of colostrum.
\end{abstract}

Keywords: calves, dairy cattle, animal health, passive immunity.

\footnotetext{
1 Recibido: 15 de julio, 2014. Aceptado: 9 de setiembre, 2014. Este trabajo pertenece al proyecto de investigación No. 737-A9-184, inscrito en la Vicerrectoría de Investigación, Universidad de Costa Rica.

2 Universidad de Costa Rica, Facultad de Ciencias Agroalimentarias, Estación Experimental Alfredo Volio Mata. San José, Costa Rica. jorge. elizondosalazar@ucr.ac.cr
} 


\section{INTRODUCCIÓN}

Las terneras recién nacidas dependen de la absorción de las inmunoglobulinas (Igs) presentes en el calostro dentro de las primeras horas de vida para protegerse contra enfermedades infecciosas en la etapa temprana de vida (Godden et al., 2012), este proceso es conocido como transferencia de inmunidad pasiva. Además de Igs, el calostro provee al neonato carbohidratos, grasas y proteínas que funcionan como combustible metabólico; también aporta vitaminas y minerales que trabajan como cofactores en procesos enzimáticos y en el mantenimiento de las funciones generales del organismo (Morril et al., 2012). El calostro también es una fuente importante de leucocitos y factores bioactivos, como la insulina, y el factor similar a la insulina I y II que afectan el desarrollo gastrointestinal posnatal (Blum y Baumrucker, 2008; Nonnecke et al., 2012).

Una variedad de factores afectan la transferencia de inmunidad pasiva, entre los más importantes se encuentran el tiempo que transcurre desde que la ternera nace hasta que consume el calostro y la masa de Igs consumida, que a su vez se ve afectada por el volumen consumido y la concentración de Igs presentes en el mismo (Godden et al., 2009 a,b).

Adecuadas concentraciones séricas de Igs entre las 24 y 48 horas de vida se han asociado con una disminución en la morbilidad y mortalidad en el periodo predestete, mejora en la ganancia de peso, edad reducida al primer parto y mayor producción de leche en la etapa de lactancia (Weaver et al., 2000; Faber et al., 2005).

Para estimar la concentración de Igs en el calostro fresco, Fleenor y Stott (1980) desarrollaron inicialmente una ecuación de regresión a partir de la gravedad específica del calostro: $\mathrm{Y}=254,716 \mathrm{X}-$ 261,451 $(r=0,84)$ (donde $Y$ es la concentración de Igs (\%) y X la gravedad específica). Dichos autores luego desarrollaron un calostrómetro que incorpora la relación entre la gravedad específica y la concentración de Igs $(\mathrm{mg} / \mathrm{ml})$ en el calostro.

El uso del calostrómetro permite estimar la calidad de calostro antes de ser suministrado a las terneras, y evitar así, un fracaso en la transferencia de la inmunidad pasiva por el uso de un calostro de baja calidad. Un aspecto importante es que la lectura del calostrómetro depende altamente de la temperatura del calostro. Mechor et al. (1991) llevaron a cabo un estudio cuyo objetivo fue investigar el efecto de la temperatura en lecturas de calostrómetro para estimar la concentración de inmunoglobulinas en el calostro bovino. Dicho estudio determinó que las lecturas difirieron en $0,8 \mathrm{mg} / \mathrm{ml}$ por cada grado centígrado en el cambio de la temperatura, por lo que la lectura debe hacerse cuando el calostro se encuentra entre 20 y $25^{\circ} \mathrm{C}$.

Durante los últimos días de gestación, grandes cantidades de Igs son transferidas de la glándula mamaria al calostro (Larson et al., 1980). Sin embargo, muchos factores pueden influir sobre la concentración de Igs en el calostro de vacas lecheras, entre ellos están la raza, el número de parto, la vacunación y el largo del periodo seco (Weaver et al., 2000; Baumrucker et al., 2010; Morril et al., 2012).

Pese a conocer su importancia, en Costa Rica no existen estudios relacionados con la concentración de Igs presentes en el calostro producido por las vacas lecheras. Por esta razón, el objetivo del presente estudio fue determinar el efecto que la raza de vaca lechera y el número de parto pueden tener sobre la concentración de inmunoglobulinas totales en el calostro.

\section{MATERIALES Y MÉTODOS}

\section{Ubicación y características de las fincas participantes}

Los datos presentados en el presente estudio corresponden a medidas de concentración de inmunoglobulinas totales, determinadas por medio de un calostrómetro (Biogenics, Oregon) en 537 muestras, obtenidas en el periodo comprendido entre los años 2010 y 2012, durante los meses de agosto a noviembre en cincuenta fincas lecheras ubicadas en las provincias de San José, Alajuela, Heredia y Cartago. Las fincas se visitaron aleatoriamente cada semana durante los meses mencionados; no existió una metodología para la escogencia de las fincas, prácticamente se utilizó cualquier lechería que estuviera dispuesta a permitir que se tomara una muestra de calostro de las vacas recién paridas al momento de la visita. Todas las lecherías eran especializadas, el ordeño se hacía en salas y de forma mecánica. Las razas de las vacas de las que se obtuvo calostro se clasificaron en Holstein, Jersey, cruce 
Holstein $\times$ Jersey y otras (para cualquier raza que no se contempla en ninguna categoría de las anteriores) y el tamaño de los hatos varió entre 10 y 300 vacas en ordeño. Estas se encontraban bajo sistemas de pastoreo continuo o en sistemas semiestabulados.

\section{Toma de muestras de calostro}

El número de muestras de calostro tomadas de cada finca fue de manera aleatoria y se basó en la disponibilidad de vacas recién paridas al momento de la visita. Las muestras fueron tomadas de forma individual y se valoraron de manera inmediata. Se utilizaron muestras de calostro de primer ordeño, y solamente aquellas donde se tuviera la certeza de que las crías no se amamantaron de la vaca antes de tomar la muestra. Estas se tomaron dentro de las primeras cinco horas posparto. Se elaboró una hoja electrónica donde se registró el nombre y localización de la finca, la identificación, la raza y el número de parto de la vaca, lo mismo que la concentración de inmunoglobulinas del calostro. Se consideró un calostro de buena calidad cuando la concentración de inmunoglobulinas fue $\geq 50 \mathrm{mg} / \mathrm{ml}$ (Fleenor y Stott, 1980; Shearer et al., 1992).

\section{Análisis estadístico}

Los datos se analizaron por medio del procedimiento MIXED de SAS (SAS Institute, 2004) para determinar la significancia de los efectos de la raza y el número de lactancia sobre la concentración de inmunoglobulinas (g/l) en el calostro, donde la vaca se consideró como la variable aleatoria. La comparación entre medias se realizó mediante la prueba de WallerDuncan $(\mathrm{P}<0,05)$.

El modelo estadístico utilizado fue:

$$
\mathrm{Y}_{\mathrm{ijk}}=\mu+\mathrm{T}_{\mathrm{i}}+\mathrm{W}_{\mathrm{j}}+(\mathrm{TW})_{\mathrm{ij}}+\operatorname{Vaca}_{\mathrm{k}}+\mathrm{e}_{\mathrm{ijk}}
$$

Donde:

$\mathrm{Y}_{\mathrm{ijk}}=$ concentración de inmunoglobulinas $(\mathrm{mg} / \mathrm{ml})$ en el calostro.

$\mu=$ media general.

$\mathrm{T}_{\mathrm{i}}=$ efecto de la raza .

$\mathrm{W}_{\mathrm{j}}=$ efecto del número de lactancia.

$(\mathrm{TW})_{\mathrm{ij}}=$ efecto de la interacción de la raza por el número de lactancia.
Vaca $=$ efecto aleatorio de la vaca.

$\mathrm{e}_{\mathrm{ijk}}=$ efecto residual.

\section{RESULTADOS Y DISCUSIÓN}

La concentración de inmunoglobulinas totales en 537 muestras de calostro varió entre 10 y 140 $\mathrm{mg} / \mathrm{ml}$, con un promedio de $85 \mathrm{mg} / \mathrm{ml}$. Del total de muestras analizadas, un 13,2\% (71/537) presentaron una concentración inadecuada de $\operatorname{Igs}(\leq 50 \mathrm{mg} / \mathrm{ml})$.

De acuerdo con Stott et al. (1981) y Petrie (1984), la concentración de inmunoglobulinas en el calostro es altamente variable entre vacas. En un estudio realizado por Shearer et al. (1992) en los Estados Unidos, de 2045 muestras de calostro analizadas, sólo 6,7\% de ellas contenían concentraciones adecuadas de Igs $(\geq$ $50 \mathrm{mg} / \mathrm{ml})$, mientras que $13,5 \%$ presentaron valores intermedios y la mayoría $(79,8 \%)$ bajos. En un estudio más reciente llevado a cabo también en los Estados Unidos, de un total de 827 muestras de calostro, 29,4\% $(243 / 827)$ no presentaron una concentración adecuada de Igs (Morril et al., 2012).

La concentración de Igs promedio de las muestras analizadas en el presente ensayo fue mayor a la reportada por Kehoe et al. (2007) (34,9 $\pm 12,23 \mathrm{mg} /$ $\mathrm{ml})$ y por Chigerwe et al. (2008) $(68,5 \pm 32,4 \mathrm{mg} / \mathrm{ml})$ pero inferior a la reportada por Bielmann et al. (2010) $(94,4 \mathrm{mg} / \mathrm{ml})$.

En el presente estudio, la temperatura del calostro se determinó por medio de un termómetro y todas las lecturas del calostrómetro se tomaron entre 20 y $25^{\circ} \mathrm{C}$.

\section{Efecto de la raza sobre la concentración de inmunoglobulinas totales}

Al considerar el efecto de raza sobre la calidad del calostro, no se encontraron diferencias significativas $(\mathrm{P}>0,05)$ (Cuadro 1). En Costa Rica ningún estudio ha valorado el efecto de la raza sobre la concentración de Igs en el calostro. En los Estados Unidos, Muller y Ellinger (1981) al comparar la concentración de Igs en el calostro de vacas de cinco razas de ganado lechero, encontraron que el promedio de Igs totales fue de 81, 66, 63, 56 y 96 mg/ml para la raza Ayrshire, Pardo suizo, Guernsey, Holstein y Jersey, respectivamente. Los autores no discutieron el por qué de las diferencias encontradas. Los resultados obtenidos aun siendo significativos no 
Cuadro 1. Efecto de la raza de la vaca sobre la concentración de inmunoglobulinas en 537 muestras de calostro provenientes de cincuenta fincas lecheras en Costa Rica. 2012.

\begin{tabular}{lrcc}
\hline Raza & n & Igs $(\mathbf{m g} / \mathbf{m l})$ & EEM \\
\hline Holstein & 270 & 88,8 & 1,9 \\
Holstein x Jersey & 64 & 85,9 & 2,5 \\
Jersey & 146 & 85,5 & 2,6 \\
Otra & 57 & 85,6 & 4,2 \\
\hline
\end{tabular}

n=número de animales, Igs=Inmunoglobulinas, EEM=Error estándar de la media.

deben generalizarse, ya que en el estudio se utilizó un número muy limitado de muestras por cada raza. En otro estudio más reciente, Morril et al. (2012) determinaron que la concentración de Igs en el calostro proveniente de vacas de la raza Holstein no difirió significativamente del calostro proveniente de vacas de la raza Jersey $(74,2$ vs. $65,8 \mathrm{mg} / \mathrm{ml}$, respectivamente).

\section{Efecto del número de parto sobre la concentración de inmunoglobulinas totales}

Al considerar el efecto del número de parto de la vaca sobre la concentración de Igs del calostro, se encontraron diferencias significativas $(p<0,05)$ (Cuadro 2) y se observó que la concentración de Igs se incrementó al aumentar el número de parto. El mismo comportamiento fue reportado por Morril et al. (2012) e indicaron que en vacas con $\geq 3$ partos la concentración de Igs fue en promedio de $95,9 \mathrm{mg} /$ $\mathrm{ml}$. Otros estudios, al igual que en esta investigación, han demostrado que la concentración de Igs en el calostro aumentó linealmente con el número de partos hasta llegar al cuarto, momento en el que se estabilizó (Devery y Larson, 1983; Robinson et al., 1988).

Se ha sugerido que el calostro producido por animales de primer parto (novillas), generalmente tiene una concentración menor de Igs que el producido por vacas con mayor número de partos. Una de las razones es que las novillas han sido expuestas a antígenos por menor tiempo que vacas con más lactancias. Además, el menor desarrollo mamario que presentan las novillas en los primeros partos podría generar un reducido mecanismo de transporte de Igs hacia la glándula mamaria, comparado con el de las vacas adultas (Moore et al., 2005; Gulliksen et al., 2008; Kehoe et al., 2011).

Con base en la información recolectada en el presente estudio, se ha podido determinar que la concentración de Igs del calostro producido por las vacas lecheras en Costa Rica es adecuada; sin embargo, es interesante rescatar que los últimos estudios que se han publicado referentes a la transferencia de

Cuadro 2. Efecto del número de parto de la vaca sobre la concentración de inmunoglobulinas en 537 muestras de calostro provenientes de cincuenta fincas lecheras en Costa Rica. 2012.

\begin{tabular}{lccc}
\hline Número de parto & $\mathbf{n}$ & Igs $(\mathbf{m g} / \mathbf{m l})$ & EEM \\
\hline 1 & 133 & $69,5 \mathrm{~d}$ & 2,7 \\
2 & 113 & $78,5 \mathrm{c}$ & 3,1 \\
3 & 100 & $84,5 \mathrm{bc}$ & 3,2 \\
4 & 85 & $98,4 \mathrm{a}$ & 3,5 \\
$\geq 5$ & 106 & $90,7 \mathrm{ab}$ & 3,2 \\
\hline
\end{tabular}

n= número de animales, $\mathrm{Igs}=$ inmunoglobulinas, $\mathrm{EEM}=$ error estándar de la media.

Diferente letra en una misma columna difieren estadísticamente, $\mathrm{P}<0,05$. 
inmunidad pasiva en terneros y terneras de lechería, indican que alrededor del $40 \%$ de estas presentan una falla en la transferencia de inmunidad pasiva (Sánchez et al., 2012; Benavides et al., 2013; Vargas et al., 2014), situación que podría deberse a factores de manejo como el volumen del calostro consumido por las terneras y el tiempo que transcurre desde el nacimiento hasta que se les ofrece el calostro.

\section{AGRADECIMIENTOS}

El autor desea expresar su más sincero agradecimiento a los estudiantes que de una $\mathrm{u}$ otra forma colaboraron con las visitas a las fincas y los muestreos de calostro. A los dueños de las fincas y a sus colaboradores por habernos permitido ingresar y tomar muestras de calostro. El agradecimiento es también extensivo al Programa de Transferencia Tecnológica de la Cooperativa de Productores de Leche Dos Pinos R.L. por la colaboración brindada durante la ejecución de la presente investigación.

\section{LITERATURA CITADA}

Baumrucker, C.R., A.M. Burkett, A.L. Magliaro-Macrina, y C.D. Dechaw. 2010. Colostrogenesis: mass transfer of immunoglobulin $\mathrm{G}_{1}$ into colostrum. J. Dairy Sci. 93:3031-3038.

Benavides, D., J.A. Elizondo, y G. Gonzalez. 2013. Estado inmunológico de terneras y terneros de lechería en la región Huetar Norte de Costa Rica. Año II. Agron. Mesoam. 24:285-291.

Bielmann, V., J. Gillan, N.R. Perkins, A.L. Skidmore, S. Godden, y K.E. Leslie. 2010. An evaluation of Brix refractometry instruments for measurement of colostrum quality in dairy cattle. J. Dairy Sci. 93:3713-3721.

Blum, J.W., y C.R. Baumrucker. 2008. Insulin-like growth factors (IGFs), IGF binding proteins, and other endocrine factors in milk: Role in the newborn. En: Z. Bösze, editor, Bioactive components of milk. Springer, NY, USA. p. 397-422.

Chigerwe, M., J.W. Tyler, J.R. Middleton, J.N. Spain, J.S. Dill, y B.J. Steevens. 2008. Comparison of four methods to assess colostral IgG concentration in dairy cows. J. Am. Vet. Met. Assoc. 233:761-766.
Devery, J.E., y B.L. Larson. 1983. Age and previous lactations as factors in the amount of bovine colostral immunoglobulins. J. Dairy Sci. 66:221-226.

Faber, S.N., N.E. Faber, T.C. McCauley, y R.L. Axe. 2005. Effects of colostrum ingestion on lactational performance. Prof. Anim. Sci. 21:420-425.

Fleenor, W.A., y G.H. Stott. 1980. Hydrometer test for estimation of immunoglobulin concentration in bovine colostrum. J. Dairy Sci. 63:973-977.

Godden, S.M., D. M.Haines, y D. Hagman. 2009a. Improving passive transfer of immunoglobulins in calves. I: Dose effect of feeding a commercial colostrum replacer. J. Dairy Sci. 92:1750-1757.

Godden, S.M., D.M. Haines, K. Konkol, y J. Peterson. 2009b. Improving passive transfer of immunoglobulins in calves. II: Interaction between feeding method and volume of colostrum fed. J. Dairy Sci. 92:1758-1764.

Godden, S.M., D.J. Smolenski, M. Donahue, J.M. Oakes, R. Bey, S. Wellsa, S. Sreevatsan, J. Stabel, y J. Fetrow. 2012. Heat-treated colostrum and reduced morbidity in preweaned dairy calves: Results of a randomized trial and examination of mechanisms of effectiveness. J. Dairy Sci. 95:4029-4040.

Gulliksen, S.M., K.I. Lie, L. Solverod, y O. Osteras. 2008. Risk factors associated with colostrums quality in Norwegian dairy cows. J. Dairy Sci. 91:704-712.

Kehoe, S.I., A.J. Heinrichs, M.L. Moody, C.M. Jones, y M.R. Long. 2011. Comparison of immunoglobulin G concentrations in primiparous and multiparous bovine colostrum. Prof. Anim. Sci. 27:176-180.

Kehoe, S.I., B.M. Jayarao, y A.J. Heinrichs. 2007. A survey of bovine colostrum composition and colostrum management practices on Pennsylvania dairy farms. J. Dairy Sci. 90:4108-4116.

Larson, B.L., H.L. Heary, y J.E. Devery. 1980. Immunoglobulin production and transport by the mammary gland. J. Dairy Sci. 63:665-671.

Mechor, G.D., Y.T. Grohn, y R.J. Van Saun. 1991. Effect of temperature on colostrometer readings for estimation of immunoglobulin concentration in bovine colostrum. J. Dairy Sci. 74:3940-3943.

Moore, M., J.W. Tyler, M. Chigerwe, M.E. Dawes, y J.R. Middleton. 2005. Effect of delayed colostrums collection on colostral $\mathrm{IgG}$ concentration in dairy cows. J. Am. Vet. Med. Assoc. 226:1375-1377.

Morrill, K.M., E. Conrad, A. Lago, J. Campbell, J. Quigley, y H. Tyler. 2012. Nationwide evaluation of quality and 
composition of colostrum on dairy farms in the United States. J. Dairy Sci. 95:3977-4005.

Muller,L.D.,y D.K.Ellinger. 1981.Colostral immunoglobulin concentrations among dairy breeds of dairy cattle. J. Dairy Sci. 64:1727-1730.

Nonnecke, B.J., W.R. Waters, J.P. Goff, y M.R. Foote. 2012. Adaptive immunity in the colostrum-deprived calf: Response to early vaccination with Mycobacterium bovis strain bacile Calmette Guerin and ovalbumin. J. Dairy Sci. 95:221-239.

Petrie, L. 1984. Maximizing the absorption of colostral immunoglobulins in the newborn dairy calf. Vet. Rec. 114:157-163.

Robinson, J.D., G.H. Stott, y S.K. Denise. 1988. Effects of passive immunity on growth and survival in the dairy heifer. J. Dairy Sci. 71:1283-1287.

Sánchez, J., J.A. Elizondo, y G. Arroyo. 2012. Estado inmunológico de terneras y terneros de lechería en la región Huetar Norte de Costa Rica. Año I. Agron. Mesoam. 23:321-327.
SAS Institute. 2004. SAS/STAT 9.1 User`s guide. Version 9.1 ed. SAS Institute Inc., Cary, N.C., USA.

Shearer, J., H.O. Mohammed, J.S. Brenneman, y T.Q. Tran. 1992. Factors associated with concentrations of immunoglobulins in colostrum at the first milking post-calving. Prevent. Vet. Med. 14:143-154.

Stott, G.H., W.A. Fleenor, y W.C. Kleese. 1981. Colostral immunoglobulin concentration in two fractions of first milking postpartum and five additional milkings. J. Dairy Sci. 64:459-465.

Vargas, O., J.A. Elizondo, y L. Noguera. 2014. Factores relacionados con la falla en la transferencia de inmunidad pasiva en terneras y terneros de lechería en la región Central Norte de Costa Rica. Nutr. Anim.Trop. 8:68-79.

Weaver, D.M., J.W. Tyler, D.C. VanMetre, D.E. Hostetler, y G.M. Barrington. 2000. Passive transfer of colostral immunoglobulins in calves. J. Vet. Intern. Med. 14:569-577. 